\title{
Getting it right first time: time to simplify our approach to the airway by using our best tools first
}

\author{
M Gibbins, TM Cook
}

Department of Anaesthesia and Intensive Care Medicine, Royal United Hospital NHS Foundation Trust, Bath, United Kingdom *Corresponding author, email: timcook007@gmail.com

Deciding on the techniques and equipment to use when managing a patient's airway during anaesthesia is a complex process. It is influenced by many factors, including the type of surgery being undertaken, patient co-morbidities, perceived risk of airway difficulty or pulmonary aspiration and the availability of more advanced equipment. While there are many guidelines for management of the unanticipated difficult airway, there are few if any for routine airway management. It is likely that current practices are heavily influenced by national and institutional norms, personal experiences and preferences of the individual anaesthetist involved.

The guidelines for management of the unanticipated difficult airway are too numerous to list but include those published by the United Kingdom's Difficult Airway Society (DAS) ${ }^{1}$ and the American Society of Anesthesiologists (ASA). ${ }^{2}$ Increasingly these guidelines concur that rescue techniques should include the use of second generation supraglottic airway devices (SGAs) and videolaryngoscopy, both during routine anaesthesia and in airway management outside of the operating theatre. ${ }^{3}$ There is growing consensus (and evidence) as to the superiority of these 'tools' over those more commonly used as first line.

So, if when we encounter difficulty, we turn to second generation SGAs and videolaryngoscopy, are we then not just losing precious time by not using them in the first place? Might the logical solution be to use our 'go to' rescue tools as our first line tools; and by doing this might we not only improve our chances of 'getting the airway right first time' but even simplify airway management to the extent that our only rescue tool becomes a scalpel?

\section{Second-generation SGAs}

SGAs have revolutionised airway management in those patients deemed not to require intubation. Since the introduction of the classic LMA (cLMA) in the late 1980s, SGAs have evolved considerably, with the development of a variety of 'upgraded' versions. Second-generation SGAs are defined as those with design features intended to reduce the risk of pulmonary aspiration. ${ }^{4}$ Common important adaptations include the addition of a drainage port and changes to improve the airway seal and prevent gastric insufflation. In addition to reducing the risk of aspiration, changes have also largely overcome some of the other limitations encountered with the first-generation devices, namely: limited peak ventilating pressure, oropharyngeal trauma and insertion difficulty. As numerous studies have shown, many devices 'work well' in over $90 \%$ of cases. ${ }^{5-8}$ Therefore, as we have largely solved the issue of efficacy, our focus should now be on safety.

Importantly, as SGAs performance and evidence base has increased, so the clinical circumstances in which they are being used have expanded. No longer are they reserved for elective surgery in spontaneously breathing patients at low risk of gastric reflux. They are now being employed in more complicated surgical procedures, such as laparoscopic surgery, ${ }^{9}$ more challenging environments, such as out of hospital cardiac arrests, ${ }^{10}$ more acute situations, such as in the rescue of a difficult airway ${ }^{1,2}$ and in more complicated patients, such as the obese or those with degrees of gastro-oesophageal reflex. Thanks to the modifications described, it is usually second-generation devices that are employed in this wide range of clinical situations, highlighting their versatility and superiority over earlier models. Importantly therefore, it is arguably the performance of SGAs in these groups of patients that we need to understand to help guide modern safe SGA use.

It is worth noting here that randomised controlled trials (RCTs) most often performed in low risk, non-obese, elective patients, exclusively managed by airway experts in university hospitals will tell us little about safety in modern usage. ${ }^{5,11}$ First, because the context of the research cannot be generalised to other settings. Second, because the studies, almost always recruiting fewer than 100 patients, are powered to examine efficacy of devices rather than safety, and rarely collect robust data on complications. By their very nature these small studies cannot exclude important rates of complications. In a study of 100 patients with no one experiencing a given complication, the upper limit of the $95 \%$ confidence interval for that complication is 3\%. Even in a study of 1000 patients with three patients experiencing the complication, the upper limit is around 1\%. Due to the low frequency of important complications in routine airway management, especially in low risk patients, safety based RCTs would simply have to be too big to be practical. A study powered to detect relevant differences in rates of pulmonary aspiration, performed in low risk starved subjects, would require several million patients. ${ }^{12,13}$ To examine safety we need to explore evidence from all available sources, including device design features and from 
benchtop, manikin and cadaveric studies where relevant. As an example, cadaveric studies - which would be unethical to repeat in live patients - have shown the effectiveness of drain ports in rapidly draining regurgitant gastric fluid and protecting the airway from aspiration for certain SGAs, thus supporting the case for the improved safety of those second generation devices. ${ }^{14}$ Another area of promise is 'real, big data' - that is, data from large series in which devices are used as they are in everyday practice, by all and sundry! This is the realm of databases, registries, and with some reservations, meta-analyses.

In this regard, it is notable to recall the results from the $4^{\text {th }}$ National Audit Project (NAP4), conducted by the Royal College of Anaesthetists (RCOA) and the DAS in the United Kingdom, which examined life-threatening complications of airway management from approximately 3 million general anaesthetics. It reported aspiration to be the commonest cause of airwayrelated anaesthesia death and specifically highlighted obese patients as of concern with regards to the use of first-generation SGAs. ${ }^{15}$ A subsequent editorial, using simple weighted scores across a number of domains measuring efficacy and safety (e.g., quality of ventilation and protection against aspiration), and using all available sources of evidence, has attempted to summarise and quantify the performance of different SGAs in various clinical settings, from the routine to the very advanced. Second generation SGAs out-performed all the earlier models, with the ProSeal LMA (PLMA, Teleflex, Athlone, Ireland) and i-gel (Intersurgical Ltd, Wokingham, UK) generating the highest total scores in most settings. ${ }^{16}$ There is also evidence (albeit graded as low quality) in the form of systematic reviews and metaanalyses of clinical trials supporting the improved efficacy of second-generation devices in both adults ${ }^{17,18}$ and children. ${ }^{6}$ Unfortunately, when evaluating measures of safety, for the reasons described above, combining efficacy-based RCTs in a meta-analysis may add little more than the individual studies.

So, we will never have high quality 'evidence' that second generation SGAs are safer than first generation alternatives but we do know their enhanced performance characteristics mean they work better, and in a wider range of settings, and the evidence 'in the round' points to enhanced safety. Despite this, several surveys in both adult and paediatric practice show that routine practice is to use a first-generation device and that this choice is most often made on the basis of cost or familiarity, and very rarely on the basis of published evidence. ${ }^{19-22}$

We are often advised 'in difficult situations to use what is familiar to us' and not use unfamiliar equipment in a pressurised setting. This is based on the belief that when we are comfortable and confident in the tools we are using, we achieve the desired outcome in as timely and safe a manner as possible. Yet, when difficulty arises, most guidelines recommend turning to a second-generation device.

The DAS guidelines recommend that "All anaesthetists should be trained to use and have immediate access to second generation SGAs". If we are going to turn to second generation SGAs in the event of a failed intubation for example, we have a duty to be comfortable and confident in their use. Therefore, using them on a regular basis must be beneficial.

\section{Videolaryngoscopy}

Turning to laryngoscopy, the device that most anaesthetists use most commonly today, the Macintosh blade, was developed in the early 1940s. It was not until well over half a century later, in 2001, that the first commercially available videolaryngoscope became widely available - the Glidescope (Verathon Inc. Bothell, United States). Even then, the intention for videolaryngoscopy was not as a replacement for direct laryngoscopy, but as a device to be used when difficulties were anticipated or encountered. In large part this is still how videolaryngoscopy is used.

On the one hand tracheal intubation using direct laryngoscopy is usually safe and without complications. On the other, complications and adverse events do occur, the overall incidence being approximately $8 \%$. Thankfully, the majority of these complications are relatively minor with no long-term sequelae - minor airway trauma occurring most frequently. ${ }^{23}$ However, intubation is awkward in approximately 1 in 17 patients ${ }^{24}$ and when difficulty arises it is unpredicted in more than $90 \%$ of cases. ${ }^{25}$ Furthermore, requiring just two or three attempts to intubate dramatically increases the incidence of important airway complications. ${ }^{26}$ The incidence of failure to intubate may vary from perhaps 1 in 2000 during routine anaesthesia to as high as 1 in 50 in the emergency department, but the consequences of failure can be devastating, and include airway trauma, awareness, cardiovascular instability, hypoxia, brain damage and death. ${ }^{27}$ Finally, it is likely that every airway death includes, during the procedure, a failure to intubate successfully, most commonly caused by failure to see the larynx adequately. ${ }^{5}$

The benefits of videolaryngoscopy are abundantly clear. These include a better view of the larynx, fewer difficult views and failed intubations, less force exerted, less airway trauma and greater ease of use. ${ }^{28}$ These findings are particularly evident in patients with a known difficult airway. Importantly, these benefits appear restricted to those who are experienced in using the devices, while those lacking experience appear to gain little or no benefit. Further, in the hands of the experienced, failure is reduced and first pass success increased..$^{29}$ In this digital era, videolaryngoscopy confers the additional advantage of the ability to store images or videos of the views obtained and techniques used to overcome difficult airways, which may be archived in patient records or used for teaching..$^{30}$ As the meta-analyses have shown heterogeneity of performance, the important research question around videolaryngoscopy should no longer be 'are they better than direct laryngoscopy', but perhaps 'which are the best devices?'

The clear benefits of videolaryngoscopy are only evident to those practised in their use. The devices, especially those with hyperangulated blades, require new techniques not only to achieve a good view, but more importantly to convert this into a prompt, first pass intubation. Again, the DAS 2015 guidelines 
state that "All anaesthetists should be trained to use, and have immediate access to, a videolaryngoscope".

Therefore, given that most difficult airways are unanticipated, that difficult intubations are associated with an increase incidence of both minor and major complications and that videolaryngoscopy has a substantial body of evidence supporting its superiority, especially in difficult airways, it seems odd that the technology has not been adopted more widely. Using videolaryngoscopy as a first line tool should provide a safety net in the event that a difficult airway is encountered. This would avoid the loss of precious time (whilst using tools we know to be inferior) and the unnecessary repeated instrumentation of the airway - a factor known to contribute to making a bad situation worse.

So, we come to the same question: as for second generation SGAs, why are they not used routinely, and should we adopt them in our everyday practice? Routine use of videolaryngoscopy appears not only useful for gaining the skills to use them when a crisis arises but actually necessary. A few hospitals have made the leap, but they are a minority. ${ }^{31}$ While the obvious barrier is cost, any cost analysis should take account of the costs of increased complications associated with an inferior technique. These include the cost of managing failure with advanced techniques, theatre delays and cancellations, admission to critical care, management of the patient's complications and finally litigation. With all these considered the cost differential is not nearly as wide as it might first appear. ${ }^{32}$

A modern, safe airway manager might make two decisions. First, they may decide to follow recognised guidelines such as the UK 2015 DAS guidelines, and second, they may decide to adopt as their first line tool for routine airway management a second-generation SGA and a videolaryngoscope. Using this approach, they will likely increase their rate of effective airway management. The range of settings where their management will succeed is likely to increase. Their incidence of failures (both failed ventilation with an SGA and failed intubation) will reduce. They will gain experience and then expertise in both techniques. Should they encounter difficulty they will already have used (expertly) the very rescue techniques that are described in the emergency guidelines. When both of these have been used optimally the next step is to proceed to an emergency front of neck airway. This approach has much to recommend it and will save considerable time and trauma compared to another operator who started with a first-generation SGA and a direct laryngoscope and was expert in neither of the newer techniques. This latter airway manager will need to attempt both the newer techniques (in which they are a novice and less likely to succeed) before declaring failure. For the anaesthetist routinely using a second-generation SGA and a videolaryngoscope the only rescue tool is most likely a scalpel. Airway management, both routine and in difficulty, has suddenly become simpler.

\section{Funding}

None

\section{Conflicts of interest}

The department has been received items of airway equipment for free or at reduced price for evaluation or research. Neither author has any financial conflicts of interest. TMC has visited several airway manufacturers to lecture but has not been paid for this.

\section{References}

1. Frerk C, Mitchell VS, McNarry AF, et al. Difficult Airway Society 2015 guidelines for management of unanticipated difficult intubation in adults. Difficult Airway Society intubation guidelines working group. Br J Anaesth. 2015;115:827-48.

2. Apfelbaum JL, Hagberg CA, Caplan RA, et al. Practice guidelines for management of the difficult airway: an updated report by the American Society of Anesthesiologists Task Force on Management of the Difficult Airway. Anesthesiology. 2013;118:251-70.

3. Higgs A, McGrath BA, Goddard C, et al. Guidelines for the management of tracheal intubation in critically ill adults. Br J Anaesth. 2018;120:323-52.

4. Cook TM, Howes B. Recent developments in efficacy and safety of supraglottic airway devices. Continuing Education in Anaesthesia Critical Care and Pain. 2011;11:56-61.

5. Cook TM. Airway complications - strategies for prevention. Anaesthesia. 2018;73:93-111.

6. Mihara T, Asakura A, Owada G, et al. A network meta-analysis of the clinical properties of various types of supraglottic airway device in children. Anaesthesia. 2017;72:1251-64.

7. Theiler L, Gutzmann M, Kleine-Brueggeney M, et al. i-gel ${ }^{T M}$ supraglottic airway in clinical practice: a prospective observational multicentre study. $\mathrm{Br} J$ Anaesth. 2012;109: 990-5.

8. Cook TM, Gibbison B. Analysis of 1000 consecutive uses of the ProSeal laryngeal mask airway by one anaesthetist at a district general hospital. Br J Anaesth 2007;99:436-9.

9. Yoon SW, Kang $\mathrm{H}$, Choi GJ, et al. Comparison of supraglottic airway devices in laparoscopic surgeries: A network meta-analysis. J Clin Anesth. 2019;55:52-66.

10. Benger JR, Kirby K, Black S, et al. Effect of a Strategy of a Supraglottic Airway Device vs Tracheal Intubation During Out-of-Hospital Cardiac Arrest on Functional Outcome: The AIRWAYS-2 Randomized Clinical Trial. JAMA. 2018;320:779-91.

11. Cook TM, Duggan LV, Kristensen MS. In search of consensus on ethics in airway research. More than caring? Anaesthesia. 2017;72:1175-9.

12. Hanley JA, Lippman-Hand $A$. If nothing goes wrong, is everything all right? Interpreting zero numerators. JAMA. 1983;249:1743-5.

13. Newman TB. If almost nothing goes wrong, is almost everything all right? Interpreting small numerators. JAMA. 1995;274:1013.

14. Schmidbauer W, Bercker S, Volk T, Bogusch G, Mager G, Kerner T. Oesophageal seal of the novel supralaryngeal airway device I-Gel in comparison with the laryngeal mask airways Classic and ProSeal using a cadaver model. Br J Anaesth. 2009;102:135-9.

15. Cook TM, Woodall N, Frerk C. Major complications of airway management in the UK: results of the 4th National Audit Project of the Royal College of Anaesthetists and the Difficult Airway Society. Part 1 Anaesthesia. Br J Anaesth. 2011;106:617-31.

16. Cook TM, Kelly FE. Time to abandon the 'vintage' laryngeal mask airway and adopt second-generation supraglottic airway devices as first choice. $\mathrm{Br} J$ Anaesth. 2015;115:497-9.

17. de Montblanc J, Ruscio L, Mazoit JX, Benhamou D. A systematic review and meta-analysis of the i-gel ${ }^{\circledR}$ vs laryngeal mask airway in adults. Anaesthesia. 2014;69:1151-162.

18. Qamarul Hoda M, Samad K, Ullah H. ProSeal versus Classic laryngeal mask airway (LMA) for positive pressure ventilation in adults undergoing elective surgery. Cochrane Database Syst Rev. 2017; 7: Art. No.: CD009026.

19. Gregory T, Golding J, Cranshaw J. A national survey of single-use and reusable laryngeal mask use in England. Eur J Anaesthesiol. 2008;25:432-4.

20. Woodall NM, Cook TM. National census of airway management techniques used for anaesthesia in the UK: first phase of the Fourth National Audit Project at the Royal College of Anaesthetists. Br J Anaesth. 2011;106:266-71.

21. Bradley AE, White MC, Engelhardt T, Bayley G, Beringer RM. Current UK practice of pediatric supraglottic airway devices - a survey of members of the Association of Paediatric Anaesthetists of Great Britain and Ireland. Paediatr Anaesth 2013;23:1006-9.

22. Engelhardt T, Virag K, Veyckemans F, Habre W; APRICOT Group of the European Society of Anaesthesiology Clinical Trial Network. Airway management in paediatric anaesthesia in Europe-insights from APRICOT (Anaesthesia Practice In Children Observational Trial): a prospective multicentre observational study in 261 hospitals in Europe. Br J Anaesth. 2018;121:66-75.

23. Hirsch IA, Reagan JO, Sullivan N. Complications of direct laryngoscopy. A prospective analysis. Anesthesiol Rev. 1990;17:34-40. 
24. Shiga T, Wajima Z, Inoue T, Sakamoto A. Predicting difficult intubation in apparently normal patients: a meta-analysis of bedside screening test performance. Anesthesiology. 2005;103:429-37.

25. Nørskov AK, Rosenstock CV, Wetterslev J, Astrup G, Afshari A, Lundstrøm LH. Diagnostic accuracy of anaesthesiologists' prediction of difficult airway management in daily clinical practice: a cohort study of 188064 patients registered in the Danish Anaesthesia Database. Anaesthesia. 2015;70:272-81.

26. Mort TC. Emergency tracheal intubation: complications associated with repeated laryngoscopic attempts. Anesth Analg. 2004;99:607-13.

27. Cook TM, MacDougall-Davis SR. Complications and failure of airway management. Br J Anaesth. 2012:109(S1):i68-i85.

28. Lewis SR, Butler AR, Parker J, Cook TM, Smith AF. Videolaryngoscopy versus direct laryngoscopy for adult patients requiring tracheal intubation. Cochrane Database Syst Rev. 2016;11:Art. No.: CD011136.
29. Pieters BMA, Maas, EHA, Knape JTA, van Zundert AAJ. Videolaryngoscopy vs. direct laryngoscopy use by experienced anaesthetists in patients with known difficult airways: a systematic review and meta-analysis. Anaesthesia. 2017;72:1532-41.

30. Zaouter C, Calderon J, Hemmerling TM. Videolaryngoscopy as a new standard of care. Br J Anaesth. 2015;114:181-3.

31. Cook TM, Boniface NJ, Seller C, et al. Universal videolaryngoscopy: a structured approach to conversion to videolaryngoscopy for all intubations in an anaesthetic and intensive care department. Br J Anaesth. 2018;120:173-80.

32. Caldiroli $D$, Byrne AJ. Cognitive re-engineering after a 15-year experience with routine videolaryngoscopy. Br J Anaesth. 2019;122:e57-e58. 